\title{
Effect of a Novel Segment Algorithm on Radiologist's Diagnosis of Masses Using Breast Ultrasound Imaging
}

\author{
Jia-Wei Tian $^{\text {ab }}$ Chun-Ping Ning ${ }^{\mathrm{b}}$ Yan-Hui Guo ${ }^{\mathrm{a}}$ Xiang-Long Tang ${ }^{\mathrm{a}}$ Ying \\ Wang $^{b}$ H.D. Cheng \\ ${ }^{a}$ School of Computer Science and Technology, Harbin Institute of Technology, Harbin, \\ 150001, China \\ ${ }^{\mathrm{b}}$ Second Hospital affiliated to the Harbin Medical University, Harbin, 150086, China \\ ${ }^{\mathrm{c}}$ Department of Computer Science, Utah State University, Utah. U.S.A
}

\begin{abstract}
To investigate the effect of using a novel segment algorithm on radiologists' sensitivity and specificity for discriminating malignant masses from benign masses using breast ultrasound (BUS) imaging. 510 conventional BUS images acquired from 109 masses were processed by the novel segment algorithm. Five radiologists were invited to analyze the original and computerized images independently according to the Breast Imaging Reporting and Data System for Ultrasound (BI-RADS-US). The diagnostic sensitivity and specificity were calculated for each radiologist by means of a binary outcome using ROC. As a result, the proposed segment algorithm can highly improve the diagnosis performance of the radiologists by meliorating the margin of the mass and the contrast of the image.
\end{abstract}

Key words: breast, mass, segment algorithm, US, BI-RADS

\section{Introduction}

Fundation: Supported, in part, by

National Natural Science Foundation of

China No. 30670546 and No. 60873142

Corresponding Author: Xianglong-Tang
Breast cancer is one of the major causes of death for female in the world ${ }^{[1,2]}$ with an increasing trend. Early detection and effective treatment are very important to the prognosis. Ultrasonography, with merits of safe and low cost, has been a preferred method both for doctors and patients $^{[3]}$. However, several inherent disadvantages of US examinations, such as speckle, clutter, acoustic shadowing, and acoustic enhancement, may compromise image quality and may be partly responsible for the inconsistent and inaccurate interpretation of BUS images.

Groups of researchers have been developed methods for computerized characterization of masses using twodimensional (2D) BUS images, and the results have shown that radiologists' accuracy in distinguishing malignant from benign masses can be significantly improved when they use a computeraided diagnosis (CAD) system as a second opinion[4,5]. In this paper, we proposed a novel segment algorithm based on homogeneity histogram, and the purpose of this study was to investigate the effect of the segment algorithm on radiologists' capability discriminating malignant masses from benign masses using BUS images, and using histological analysis as the reference standard. 


\section{Materials and Methods}

The study group consisted of 97 women with 109 solid masses who had undergone 2D BUS examination between 2005 and 2007. The mean age was 41.7 years, range 28 83 years. Diameters of the masses ranged from $0.8 \mathrm{~cm}$ to $2.6 \mathrm{~cm}$. The nature of all masses was confirmed with histopathology examination. Among the 109 masses, 55 were benign including 33 fibroadenomas, 16 introductal papillomas and 6 lipomas; 54 were malignant including 41 infiltrative ductal carcinomas, 6 invasive lobular carcinomas, 4 simplex carcinomas, 1 inflammatory carcinoma and 2 medullary carcinomas.

Totally 510 images were obtained from the 109 masses with high-resolution Vivid 7 sonography system (GE Healthcare, Milwaukee, WI) equipped with $7.5 \sim 14 \mathrm{MHz}$ linear transducer. The examination was performed by a breast radiologist who has 27 years of experience in BUS. In each case, at least three static images including radial and antiradial images were acquired. The average size of the image is $450 \times 450$ pixels. All the 510 images were processed by the segment algorithm (see appendix), and the processed images were saved in a folder, while the original images in another folder.

Five breast radiologists were invited to analyze the original and processed images independently. Each observer was provided with a sheet containing the descriptors described in the BI-RADS-US [6], and was instructed to select the most appropriate description for each lesion including both conventional and processed sonograms. Also, readers provided an assessment category to indicate the probability of malignancy according to the BI-RADS-US.

\section{Statistical Analysis}

The sensitivity and specificity were calculated for each radiologist by using receiver operating characteristic (ROC) analysis. Medcalc statistical software (Version 9.5.2 Schoonjans, Frank) was used for this statistical analysis.

\section{Results}

\subsection{Images}

All the 510 images were processed by the proposed segment algorithm. Compared with the original image, the processed images had higher contrast, the margin of the masses and calcification are more obvious. Fig.1 showed an infiltrative ductal carcinomas confirmed by histology. The segmented image (b) was more distinct than the original image (a), especially the calcifications, and we can see that the spicules of the mass were clearer. Based on the original image, three of the radiologists defined the margin of the mass as indistinct, one of them considered it to be angular, and the other described it as spiculate, while reading the processed images, three of the radiologists agreed it to be spiculate and the other two described it as angular.

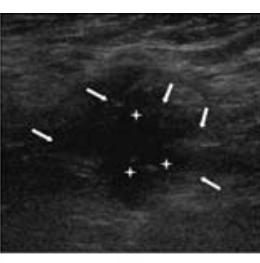

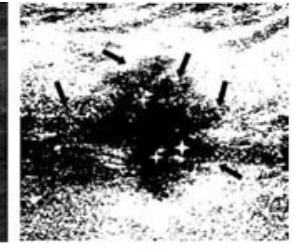

Fig.1. US of a 62-year-old woman with an invasive ductal carcinoma. (a) the original image. (b) the segmented image. ( $\rightarrow$ ) The spicule of the mass.( $($ ) The calcification of the mass.

\subsection{ROC curve}

The ROC curves of the diagnosis were shown in Fig.2. It was obvious that the ROC curve of the radiologists' diagnosis 
based on the segmented BUS images was more convex than that based on the conventional BUS images.
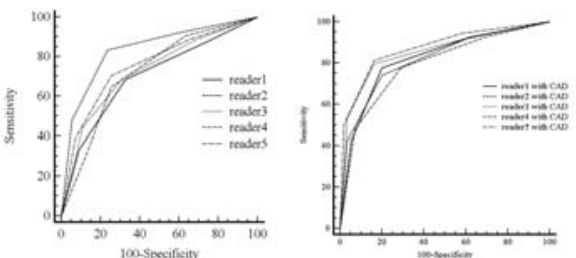

Fig.2. Roc Curve of The Conventional US with and without the segment Algorithm

Results of the reliability evaluation of the proposed segment algorithm were reported in Table 1 . For each radiologist the $\mathrm{Az}$ value and confidence interval (CI) of the ROC curve were listed, as well as the performance at the best classification accuracy, including sensitivity, specificity, positive predictive value (PPV), and negative predictive value (NPV). The $\mathrm{Az}$ values of the radiologists ranged from 0.70 to 0.84 without using the algorithm and from 0.79 to 0.88 using the segmentation algorithm (Table.1). The improvement in $\mathrm{Az}$ values was statistically significant for three of the five radiologists. Radiologist 1 had the largest $\mathrm{Az}$ value change: The $\mathrm{Az}$ value was 0.70 which changed to 0.82 using the algorithm.

The sensitivity, specificity, PPV and NPV of the five radiologists were also displayed in Table.1. To readers 1, 3, 4, 5, the entire index became higher when they analyzing the images processed by the algorithm.

\section{Discussion}

Being an assistant to doctors, computeraided diagnosis (CAD) is a novel method in detecting early breast cancer, which may greatly improve image quality. Segmentation is an essential and critical step for detecting lesions and making correct diagnosis. Several algorithms have been proposed ${ }^{[7,8]}$.

In this study, we proposed a novel segment algorithm based on homogeneity histogram. Five readers described the specific US findings of solid breast masses and provided a BI-RADS-US final assessment category based on the images before and after process by the segment algorithm. As a result, the internal echo of the masses, especially the calcification could be displayed better, and the margin of the masses was more clear and distinct, especially the spicule signs. The diagnostic performance of the five radiologists was significantly improved when they analyzed the processed images.

In conclusion, the proposed CAD algorithm can improve the diagnosis performance of the radiologist by meliorating the internal echo of the masses and the contrast of the image.

This study also has two additional weaknesses. First, the observer are much more familiar to the original images, most of them are not familiar with processed images, which would theoretically have affected their choices. Second, this algorithm is not sensitive enough to the shadow of the masses, and some of the masses with acoustic shadow and the masses near the nipple can't be segmented correctly.

\section{Appendix}

Breast ultrasound images have some degree of fuzziness, so preprocessing is necessary to suppress speckle noise, to enhance the contrast, and to improve the quality of the images.The preprocessing includes: 1) speckle and reduction; 2) mammary gland region detection; 3) image enhancement.

In order to remove speckle noise, we implement an algorithm based on two- 
dimension (2D) textural homogeneity histogram and directional average filters.

In this paper, a method based on stepdown threshold technique, which selects a threshold in each step, is proposed to extract the mammary gland area from the entire image.

The breast ultrasound image enhancement algorithm using fuzzy $\operatorname{logic}{ }^{[9]}$ is very effective not only in enhancing the entire image but also in enhancing the lesions features. It also makes the details of images much clearer without over-enhancement and underenhancement. The quality of mammary gland area with low contrast is improved, while the lesions in this area become clear and their edges are enhanced precisely.

After preprocessing, mammary gland region is extracted and the lesions become more distinct. We segment the mammary gland regions using eliminating particle swarm optimization (EPSO) algorithm and k-means clustering analysis.

\section{Reference}

[1] Y. Luo, J. Zhang, Y. Liu, A. C. Shaw, X. Wang, S. Wu, X. Zeng, J. Chen, Y. Gao, and D. Zheng, "Comparative proteome analysis of breast cancer and normal breast, " Molecular Biotechnolog, pp. 233 -244,2005.

[2] A. Jemal, T. Murray, E. Ward, A. Samuels, R. C. Tiwari, A. Ghafoor, E. J. Feuer, and M. J. Thun, "Cancer
Statistics, 2005," CA Cancer J Clin, vol.55, pp. 10-30, 2005.

[3] E. Paci, "Mammography and beyond: developing technologies for the early detection of breast cancer," Breast Cancer Res, vol 4, pp. 123-125, 2002.

[4] Chen CM, Chou YH, Han KC, et al. "Breast lesions on sonograms: computer-aided diagnosis with nearly setting-independent features and artificial neural networks, ” Radiology. vol.226, pp. 504-514,2003.

[5] Horsch K, Giger ML, Venta LA, Vyborny CJ. "Computerized diagnosis of breast lesions on ultrasound,"Med Phys. vol.29, pp.157-164, 2002.

[6] American College of Radiology. BIRADS: ultrasound, 1st ed. In: Breast imaging reporting and data system: BI-RADS atlas, 4th ed. Reston, VA: American College of Radiology, 2003

[7] K. Horsch, M. L. Giger, L. A. Venta, and C. J. Vyborny, "Automatic segmentation of breast lesions on ultrasound, ” Med Phys, vol.28, no.8, pp. 1652-9, 2001.

[8] K. Horsch, M. L. Giger, L. A. Venta, and C. J. Vyborny, "Computerized diagnosis of breast lesions on ultrasound, ” Med Phys. vol 29, no.2, pp. 157-64, 2002.

[9] Y. H. Guo, H. D. Cheng, J. H. Huang, J. W. Tian, W. Zhao, L. T. Sun, and Y. X. Su, "Breast ultrasound image enhancement using fuzzy logic," Ultrasound in Medicine and Biology. vol.32, no.2, pp. 237-247 2006.

Table.1. Performs of Readers in Assessing 109 Breast Masses basing on the Conventional BUS images and BUS images with using the segment algorithm

\begin{tabular}{|c|c|c|c|c|c|c|c|c|c|c|c|c|c|}
\hline \multirow[b]{2}{*}{5} & \multicolumn{3}{|c|}{$\mathrm{Az}^{*+}$} & \multicolumn{2}{|c|}{ Sensitivity } & $P$ & \multicolumn{2}{|c|}{ Specificity } & 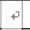 & \multicolumn{2}{|c|}{ PPV } & \multicolumn{2}{|c|}{$\mathrm{NPV}$} \\
\hline & With CAD & Without CAD & $\mathrm{P}^{\mathrm{m}} \mathrm{p}$ & With CAD & $\begin{array}{l}\text { Without } \\
\text { CAD }\end{array}$ & a & With CAD & $\begin{array}{l}\text { Without } \\
\text { CAD }\end{array}$ & . & With CAD & $\begin{array}{l}\text { Without } \\
\text { CAD }\end{array}$ & With CAD & $\begin{array}{c}\text { Without } \\
\text { CAD }\end{array}$ \\
\hline Reader1 & $0.82(0.74,0.90)$ & $0.70(0.61,0.79)$ & 0.001 & $80.00(44 / 55)^{2}$ & $67.27(37 / 55)=$ & 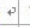 & $77.78(42 / 54)^{*}$ & $68.52(37 / 54)$ & 0 & $78.57(44 / 56)+$ & $68.52(37 / 54)=$ & $+79.24(42 / 53))^{+}$ & $67.28(37 / 55)+4$ \\
\hline Reader $2 \%$ & $0.82(0.73,0.89)=$ & $0.84(0.75,0.90)+$ & $0.648+$ & $74.55(41 / 55))^{2}$ & $76.36(42 / 55)$ & . & $79.63(43 / 54)^{4}$ & $83.33(45 / 54)$ & 0 & $78.85(41 / 52)+$ & $82.35(42 / 51)^{+}$ & o $75.44(43 / 57)^{2}$ & $77.58(45 / 58)$ \\
\hline Reader $3{ }^{\circ}$ & $0.85(0.78,0.93)$ & $0.73(0.64,0.81)$ & 0.010 & $83.64(46 / 55)^{2}$ & $69.09(38 / 55)$ & 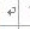 & $79.62(43 / 54)$ & $68.51(37 / 54)$ & 0 & $80.70(46 / 57)$ & $69.09(38 / 55)=$ & $82.69(43 / 52)^{+}$ & $68.52(37 / 54)$ \\
\hline Reader4 & $0.79(0.71,0.88)$ & $0.72(0.63,0.81)^{+}$ & $0.138+$ & $70.91(39 / 55)^{\circ}$ & $74.55(41 / 55)=$ & P & $77.78(42 / 54)+$ & $64.81(35 / 54)^{-7}$ & 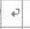 & $76.47(39 / 51)=$ & $68.33(41 / 60)=$ & $72.41(42 / 58)+$ & $71.43(35 / 49)+$ \\
\hline Reader5 & $0.88(0.81,0.94)$ & $0.76(0.67,0.84)$ & $0.001^{4}$ & $83.64(46 / 55)^{\circ}$ & $74.54(41 / 55)=$ & 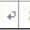 & $81.48(44 / 53)=$ & $50.00(27 / 54)$ & 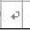 & $82.14(46 / 56)+$ & $60.29(41 / 68)^{+}$ & - $83.2(44 / 53)^{2}$ & $65.85(27 / 41)^{2}$ \\
\hline
\end{tabular}

